$$
\lambda_{\text {C.E. }}=\sqrt{1-A^{2}} \cdot|\lambda|_{\text {S.M. }}
$$

The core-excitation model requires $\sqrt{1-A^{2}}$ to be a small quantity, which reduces $\lambda_{\text {theor }}$ with regards to its shell model value. Consequently the agreement between $\lambda_{\exp }$ and $\lambda_{\text {theor }}$ would be better. Further, we may change our point of view and consider $A$ as the unknown quantity. Putting $\lambda_{\text {C.E. }}=\lambda_{\exp }$ the relationship (3) may serve as a measurement of the purity of the core-excitation state. In $\mathrm{Tl}^{203}, A^{2}=0.96$ demonstrates the validity of the model.

The same conclusions hold of course for the other odd Tl-isotopes. These conclusions may be also extended to the odd Au-isotopes, the roles of the $s_{1} \frac{1}{2}$ and $d \frac{3}{2}$ states being permuted. As a matter of fact, ${ }^{2}$ a systematical study of the internal conversion coefficient would provide a test of the validity of the de-Shalit model for this set of nuclei.

Firmly, we shall conclude with a short remark on the M1 transitions between states of the same multiplet. These transitions are proportional to $\left(g_{c}-g_{p}\right) *$. Thus, an accidental cancellation may reduce $M_{\gamma}$, whereas $M_{e}$ is not affected by this cancellation, so that large values of $\lambda$ are expected. This case is similar to the M1 transition within one rotational band, which has been studied by Reiner 7 ).

\section{References}

1) E. L. Church and J.Weneser, Ann. Rev. Nuclear Sci. $10(1960) 193$.

2) G.J.Nijgh and A.H. Wapstra, Nuclear Phys.9 (1959) 545

3) T.R.Gerholm, B.G.Petterson, B. Van Nooijen and Z. Grabowski, Nuclear Phys. 24 (1961) 177.

4) E. L. Church, A.Schwarzschild and J.Weneser, Phys Rev. 133 (1963) 35 B.

5) R.D. Lawson and J. L. Uretsky, Phys. Rev. 108 (1957) 1300 .

6) A. de-Shalit, Phys. Rev. 122 (1960) 1530.

7) A.S. Reiner, Nuclear Phys. 5 (1958) 544.

* $g_{\mathrm{c}}$ and $g_{\mathrm{p}}$ being the gyromagnetic factor of the core and of the odd proton respectively.

\title{
DETERMINATION OF NUCLEAR MATRIX ELEMENTS IN THE DECAY OF Sb124 INCLUDING FINITE NUCLEAR SIZE EFFECTS *
}

\author{
H. J. FISCHBECK and W. M. GREENBERG \\ H.M. Randell Laboratory of Physics \\ The Universily of Michigan, Ann Arbor, Michigan
}

Received 9 March 1964

It has been customary for the last few years to obtain nuclear matrix elements from experimental observables in $\beta$ decay through the use of theoretical expressions simplified by the KonopinskyUhlenbeck approximation 1). In this approximation, the finite size of the nuclear charge distribution is neglected and the electron radial wave functions, calculated for a point charge nucleus and evaluated at the nuclear radius, $R$, are expanded in terms of $R$ neglecting contributions of order $(\alpha Z)^{2}$. Here, $\alpha$ is the fine structure constant and $Z$ the nuclear charge. However, it has been shown recently 2) that an analysis of experimental data using this approximation may result in matrix element parameters which differ con-

\footnotetext{
* Work supported by the U.S.Atomic Energy Commission.
}

siderably from those obtained using more exact theoretical expressions.

We have attempted a more reliable determination of the nuclear matrix elements in the decay of \$b124 by using the theoretical expressions of first forbidden $\beta$ decay as given by Morita 3 ). Here, finite nuclear size effects are readily included since the electron radial wave functions appear explicitly. The electron radial wave functions are tabulated by Bhalla and Rose 4), who calculated these functions at the nuclear surface assuming an electrostatic potential corresponding to a uniform spherical charge distribution inside the nucleus, $r \leqslant R$, and to a point charge for $r>R$. As may be seen from table 1, the values for the nuclear matrix element parameters obtained for Sb124 by investigators using the Konopinsky-Uhlenbeck approximation differ con- 
Table 1

Comparison of nuclear matrix element parameters in the $\mathrm{Sb}^{124}$ decay.

\begin{tabular}{cccc}
\hline$Y$ & $x$ & $u$ & Ref. \\
\hline $0.60 \pm 0.30$ & $-0.05 \pm 0.10$ & $-0.06 \pm 0.14$ & 11 \\
$0.64 \pm 0.12$ & $-0.084 \pm 0.050$ & $-0.079 \pm 0.075$ & 6 \\
$1.05 \pm 0.15$ & $0.06 \pm 0.05$ & $0.15 \pm 0.05$ & 7 \\
$0.30 \pm 0.08$ & $-0.14 \pm 0.02$ & $0.13 \pm 0.03$ & 8 \\
$0.90 \pm 0.05$ & $0.60 \pm 0.05$ & $-0.025 \pm 0.025$ Set I \\
& & \multicolumn{3}{c}{ Present work } \\
$0.7 \pm 0.1$ & $-0.025 \leqslant x \leqslant 0$ & $0 \leqslant u \leqslant 0.025$ Set II
\end{tabular}

siderably from each other, while the experimental data from which these values were obtained are in fair agreement. (See figs. 1, 2 and 3, for example.) Therefore, an analysis based on more exact * theoretical expressions and taking into account all existing experimental information seemed desirable.

The transition of interest in this analysis is the outer $2.31 \mathrm{MeV} \beta$ group in the first forbidden nonunique $\beta$ decay of the $3^{-}$ground state of $\mathrm{Sb}^{124}$ to the $603 \mathrm{keV}$ first excited $2^{+}$state in $\mathrm{Te}^{124}$, followed by a $603 \mathrm{keV} \mathrm{E} 2 \gamma$ ray to the $0^{+}$ground state in $\mathrm{Te}^{124}$ (see insert of fig. 1). For this

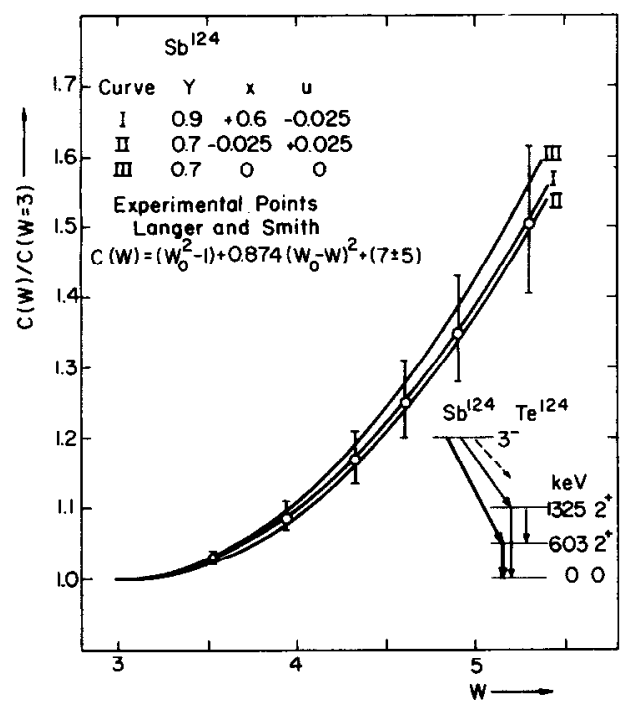

Fig. 1. The normalized spectrum shape factor of the $2.31 \mathrm{MeV} \beta$ branch in $\mathrm{Sb} 124$ as a function of the $\beta \mathrm{en}-$ ergy $\left(W\left(m_{0} c^{2}\right)\right.$. The curves are calculated from the theoretical expression for the shape factor as given in ref. 2 .

\footnotetext{
* The only remaining major approximations are the neglect of the variation of the electron wave function in the radial matrix element integration over the nuclear volume and interference terms from first and third forbidden matrix elements.
}

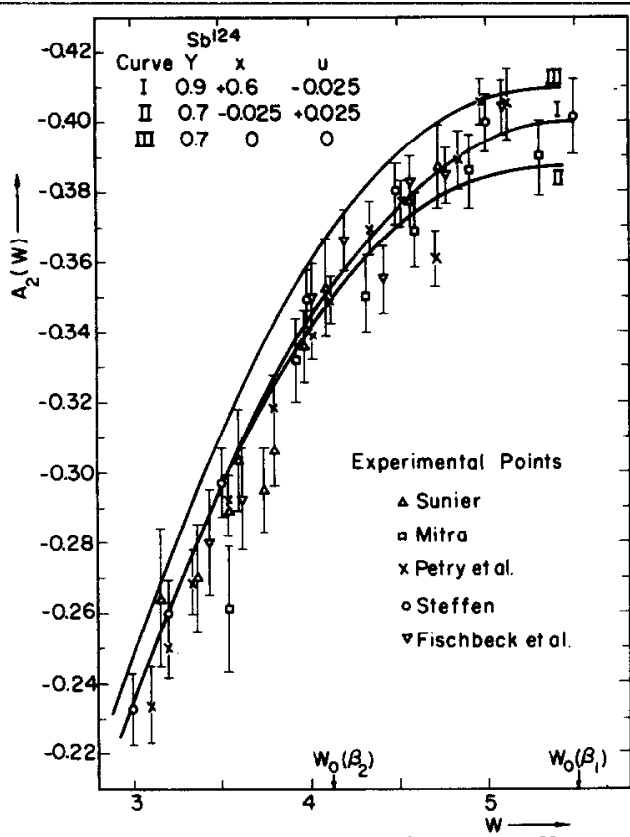

Fig. 2. The $\beta-\gamma$ directional correlation coefficient $A_{2}(W)$ of the $2.31 \mathrm{MeV}(\beta)-0.603 \mathrm{MeV}(\gamma)$ transition in Sb124 as a function of the $\beta$ energy $W\left(m_{0} c^{2}\right)$. The curves are theoretical and calculated from the expression as given in ref. 2 .

transition the spectrum shape factor was measured by Langer and Smith 5$)$ and found to be of the form $C(W)=\left(W_{0}^{2}-1\right)+0.874\left(W_{O}-W\right)^{2}+$ $+(7 \pm 5)$. The shape factor as a function of the $\beta$ energy $W$ (in units of $m_{0} c^{2}$ ) and normalized to 1 at the lowest energy, is shown in fig. 1. The $\beta-\gamma$ directional correlation coefficient $A_{2}(W)$ has been measured as a function of the $\beta$ energy by several investigators $6-10$ ). The results of these measurements are shown in fig. 2. The $\beta-\gamma$ circular polarisation correlation was measured by Alexander and Steffen 11) for several angles, $\theta$, between the $\beta$ and $\gamma$ rays at an average $\beta$ energy $W=4.6\left(m_{0} c^{2}\right)$. The degree of circular polarisation, $P_{c}(\theta, W)=\omega(p / W) \cos \theta$, where $\omega$ is the circular polarisation coefficient, is shown in fig. 3. All this experimental information was fitted with the theoretical expressions for the shape factor, $C(W)$, the $\beta-\gamma$ directional correlation coefficient, $A_{2}(W)$, and the $\beta-\gamma$ circular polarisation coefficient, $\omega(\theta, W)$. The theoretical expressions for these observables are given by Morita 3 ) in a general form and have been reformulated in ref. 2 in terms of the matrix element ratios $u=$ $\int i \sigma \times r / \int B_{i j}, x=-C_{\mathrm{V}} \int r / C_{\mathrm{A}} \cdot B_{i j}$ and $y=$ $-C_{\mathrm{V}}\left[i \alpha / C_{\mathrm{A}} B_{i j}\right.$ for a $3^{-}(\beta) 2^{+}(\gamma) 0^{+}$transition. In order to allow easy comparison with earlier results, the matrix element combination $Y=$ 


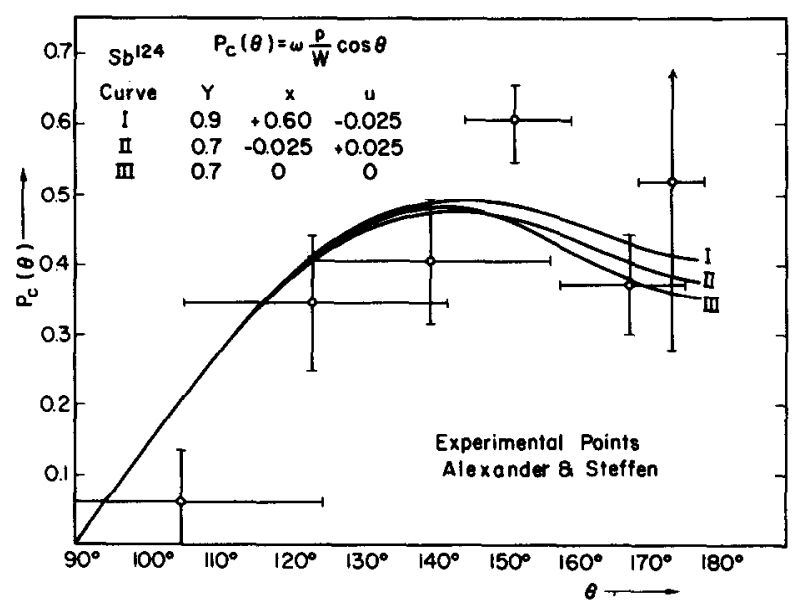

Fig. 3. Angular dependence of the circular polarisation of the $0.603 \mathrm{MeV} \gamma$-ray for an average $\beta$ energy of 4.6 $\left(m_{0} c^{2}\right)$. The theoretical curves are calculated from the expression as given in ref. 2 .

$y-\xi(u+x)$ instead of $y$ will be given here: With the aid of the University of Michigan IBM 7090 computer, a search for the values of $u, x$ and $Y$ which fit the experimental data best (i.e. have the lowest $\chi^{2}$ values) was conducted. The results are shown as curves I, II and III in figs. 1, 2 and 3. We conclude that either $Y=0.90 \pm 0.05$, $x=0.6 \pm 0.05, u=-0.025 \pm 0.025 ;$ or $Y=$ $0.7 \pm 0.1,-0.025 \leqslant x \leqslant 0,0 \leqslant u \leqslant 0.025$. The assigned error limits reflect the steepness of the $\chi^{2}$ minimum. With the first parameter combination, the goodness of fit is much more sensitive to a variation of $Y$ than is the case with the second set. It is obvious from the curves II and III in the figures, that, because of the experimental uncertainties, only an upper limit can be placed on the magnitude of the parameters $x$ and $u$ with $Y=0.7$.

From the nuclear parameters the respective nuclear matrix elements can be calculated since the $B_{i j}$ matrix element is related to the partial half-life, $t$, by

$$
\left|C_{\mathrm{A}} \int B_{i j}\right|^{2}=\frac{\pi^{3} \ln 2}{f_{c} t},
$$

where $f_{\mathrm{c}}=\int C(W) F_{\mathrm{o}}(W) p q^{2} W \mathrm{~d} W$. The partial half-life was calculated from the measured branching ratio 5) and the total half-life taking the $2.31 \mathrm{MeV} \beta$ group to be $(22 \pm 1) \%$ and the halflife of $\mathrm{Sb}^{124}$ as 60.1 days. The numerical values for the resulting nuclear matrix elements are summarized in table 2 . The coordinate type matrix elements are normalized to the nuclear radius $R=1.21 A^{1} \mathrm{fm}$, and the currently accepted
Table 2

Nuclear matrix elements in the decay of $\mathrm{Sb}^{124}$

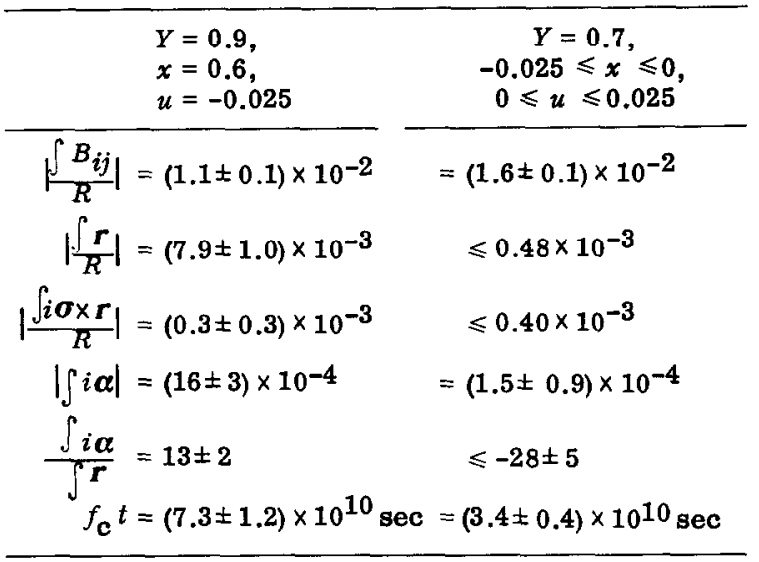

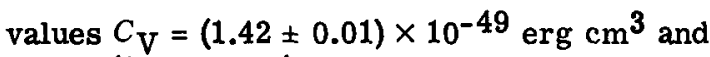
$c_{\mathrm{A}}=-(1.19 \perp 0.03) \times C_{\mathrm{V}}$ are used for this calculation.

It is interesting to note that the more exact analysis used in this investigation indicates that selection rules may indeed play an important role in the $\mathrm{Sb}^{124}$ decay, since for the second set of matrix element parameters both $x$ and $u$ are at least 30 times smaller than $Y$ which contains the large Coulomb factor $\xi=\alpha Z / 2 R=12$. The $B_{i j}$ matrix element (tensor rank 2), while considerably smaller than in unique transitions, is almost two orders of magnitude larger than the rank 1 matrix elements. Unfortunately, the accuracy of the experimental data is not good enough to allow a unique determination of the nuclear parameters. It is evident from a comparison of the experimental data with the theoretical curves that in order to resolve the two possible sets of nuclear parameters, the accuracy of the experiments would have to be improved by about a factor of 10. Therefore, considering the difficulty in achieving even the present degree of accuracy in some of these experiments, it would be useful to have more detailed theoretical estimates of the magnitude of the nuclear matrix elements 12 ).

The authors would like to thank Professor $M$. Wiedenbeck for his support and interest. Dr. R. Newsome for valuable discussions and Dr. R. Woods for his advice on the use of the computer.

\section{References}

1) T. Kotani, Phys. Rev. 114 (1953) 795

2) R.W.Newsome Jr. and H.J. Fischbeck, Phys. Rev. 133B (1964) 273

3) M. Morita and R.S. Morita, Phys. Rev. 109 (1958) 2048. 
4) C.P. Bhalla and M.E.Rose, ORNL-3207 (1961)

5) L. M. Langer and D.R.Smith, Phys. Rev. 119 (1960) 1308.

6) J.W.Sunier, Helv. Phys. Acta 36 (1963) 429.

7) S.K. Mitra, Nuclear Phys. 47 (1963) 293.

8) R.F.Petry, K.S.R.Sastry and R.G.Wilkinson, Phys. Rev., to be published.
9) R. M. Steffen, Phys, Rev. 124 (1961) 145.

10) H. J. Fischbeck and M. L. Wiedenbeck, Bull. Am. Phys. Soc. II, 6 (1961) 238.

11) P. Alexander and R. M. Steffen, Phys. Rev. 124 (1961) 150 .

12) C. S.Wu and L. Kisslinger, Bull. Am.Phys. Soc.II, 7 (1962) 539

\section{POSSIBLE FOUR-QUASIPARTICLE EXCITED STATE OF Er166 \\ Z. PREIBISZ and J. $\dot{\mathbf{Z} Y L I C Z}$ \\ Institute of Nuclear Research, Dept. of Physics, Swierk by Warsaw, Poland}

Received 12 March 1964

It is the aim of the present communication to summarize and to discuss the results of measurements in which the physical nature of $\mathrm{Er}^{166}$ high energy levels has been investigated. There are $2137 \mathrm{keV}$ and $2165 \mathrm{keV}$ excited levels established by Grigoriev et al. 1) and Harmatz et al. 2 ). As a basis for the discussion the decay scheme from the latter paper has been taken (fig. 1). On the figure some uncertain levels as well as corresponding gamma transitions from these levels are omitted. There are also introduced some changes following from the results of the present paper as well as from the previous investigations carried out in the same laboratory 3-5).

The spin of the $2137 \mathrm{keV}$ and $2165 \mathrm{keV}$ levels seems to be well established $(I=3)$ but the question of the parity has been open till now.

According to Harmitz et al. 2) the parity of the considered levels is odd and the gamma transitions between these levels and the ground state rotational band have E1 multipolarity. Grigoriev et al. 6) suggest multipolarity M2 (at least for the $2057 \mathrm{keV}$ transition from the level of $2137 \mathrm{keV}$ ), which corresponds to the same odd parity, but gives a difference of one order of magnitude in absolute internal conversion coefficients. Preibisz et al. 5) have determined $\mathrm{K}$-conversion coefficients of the 2057 and $2083 \mathrm{keV}$ transitions. For the measurements of the gamma line intensities they applied both the external conversion method and scintillation technique.

The results compared with theoretical values are presented in table 1. This comparison suggests M1 or E2 multipolarity for both transitions

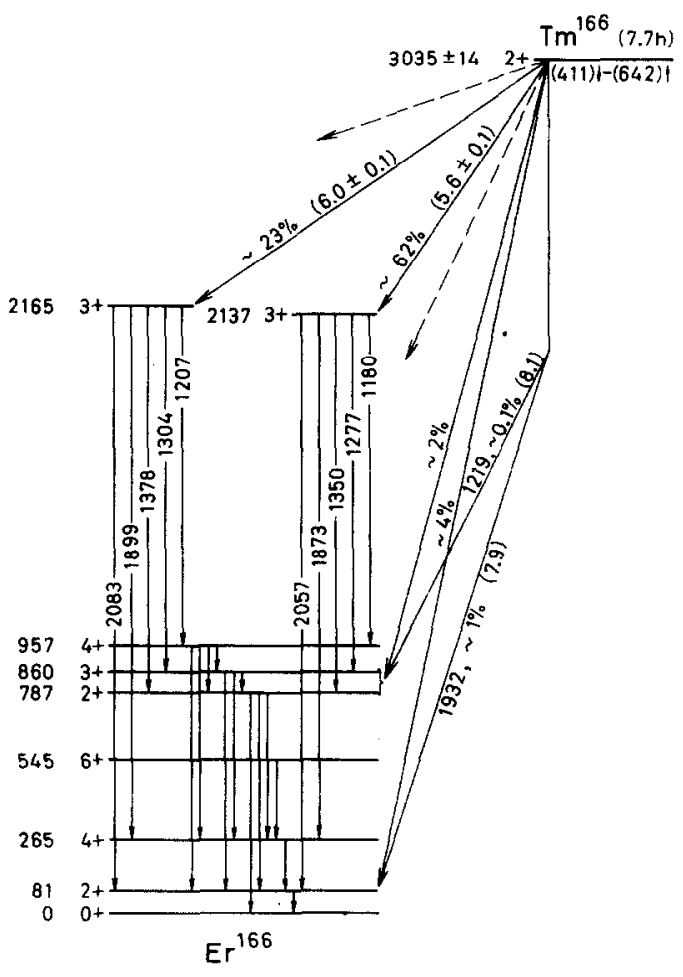

Fig. 1. The decay scheme of $\operatorname{Tm}^{166}$.

which corresponds to even parity assignment of the discussed levels.

The gamma transitions from the $2137 \mathrm{keV}$ level to the gamma vibrational band were previous- 